\title{
5. Themenbereich Stammzellen und Organoide: Chancen für die regenerative und personalisierte Medizin, Gen- und Biotechnologie $^{1}$
}

\subsection{Zur Definition von Stammzellen und Organoiden}

Stammzellen sind definiert durch zwei Eigenschaften: Stammzellen können sich erstens fast unbegrenzt teilen und vermehren (Vermehrungspotenzial), und zweitens diesen Vermehrungszustand verlassen und je nach Stammzelltyp unterschiedliche spezialisierte Zelltypen ausbilden (Differenzierungspotenzial). Mit diesen beiden Eigenschaften in ein und derselben Zelle unterscheiden sich Stammzellen von anderen Zellen in unserem Körper. So sind Stammzellen während der Embryonalentwicklung wichtig für den Aufbau der verschiedenen Organe und Gewebe in unserem Körper (humane embryonale Stammzellen, hES-Zellen) und im erwachsenen (adulten) Organismus für deren Aufrechterhaltung und Reparatur (adulte, somatische oder auch gewebespezifische Stammzellen).

Organoide sind dreidimensionale, organähnliche Zellverbände in Zellkultur, bei denen sich verschiedene Zelltypen so organisiert haben, wie es näherungsweise für das entsprechende Organ im Körper typisch ist. Sie weisen dabei drei Merkmale auf: Selbstorganisation, Vielzelligkeit und Funktionsfähigkeit. Die Bandbreite der Organe, die mit Organoiden erforscht werden können, wächst rapide an und umfasst u. a. Gehirn, Darm, Niere, Magen, Pankreas, Lunge, Leber, Prostata, Speiseröhre, Gallenblase

1 Teile dieses Textes sind entnommen aus Zenke et al. (2018a), insbesondere aus Zenke, MarxStölting, Schickl (2018b): Aktuelle Entwicklungen der Stammzellforschung: eine Einleitung, und aus Bartfeld et al. (2020a), insbesondere aus Bartfeld, Schickl, Pichl, Osterheider, Marx-Stölting (2020b): Organoide in Forschung und Anwendung: eine Einführung. 
und den weiblichen Reproduktionstrakt. Daneben kann auch der Embryo durch sogenannte Embryoide modelliert werden.

Stammzellen und Organoide werden hier gemeinsam betrachtet, da Stammzellen verwendet werden, um Organoide im Labor wachsen zu lassen. In der Kombination von Stammzell- und Organoidforschung ergeben sich neue und bisher ungeahnte Möglichkeiten für die Entwicklungsbiologie, die biomedizinische Grundlagenforschung, die Medikamentenentwicklung und regenerative Medizin sowie die Gen- und Biotechnologie. Auch haben diese z. T. revolutionären Entwicklungen einen maßgeblichen Einfluss auf den Erkenntnisgewinn über die Entwicklung von Organen und Organismen, die Modellierung von Krankheiten im Labor und die personalisierte Medizin, und stehen damit im Fokus des wissenschaftlichen, medizinischen und öffentlichen Interesses. Daraus ergeben sich wissenschaftliche, medizinische, ethische, rechtliche und gesellschaftliche Herausforderungen, die bereits jetzt absehbar sind, zeitnah adressiert werden müssen und auch einen gesellschaftlichen Diskurs erfordern.

Dieser Beitrag kann und will nicht die komplette Bandbreite der Stammzell- und Organoidforschung abbilden, sondern einige ausgewählte Stammzelltypen und Organoidmodelle vorstellen. Ziel ist, über den gegenwärtigen Sachstand zu berichten und zukünftige Entwicklungen und Perspektiven der Stammzell- und Organoidforschung aufzuzeigen.

\subsection{Stammzellen}

\subsubsection{Adulte und pluripotente Stammzellen}

Stammzellen können entsprechend ihrem Entwicklungspotenzial unterschieden werden, sowie danach, ob sie natürlich vorkommen („konventionelle Stammzellen“) oder künstlich hergestellt („engineered“) werden.

Die „konventionellen“ Stammzellen werden nach ihrem jeweiligen Entwicklungspotenzial klassifiziert: Totipotente Stammzellen der befruchteten Eizelle (Zygote) besitzen die Fähigkeit, alle Zelltypen des Embryos inklusive der extraembryonalen Zellen (Plazenta) und somit einen ganzen Organismus hervorzubringen. Nach der Befruchtung induzieren maternale (mütterliche) Faktoren in der Zygote die epigenetische Reprogrammierung der Genome von Eizelle (Oozyte) und Spermium, und etablieren so die Fähigkeit der Totipotenz. Die epigenetische Prägung von Eizelle und Spermium, d. h. die biochemischen Veränderungen der DNA und der Verpackung der DNA (Chromatin), haben einen Einfluss auf die Genregulation und die Spezialisierung der Zellen (zur Epigenetik siehe Walter/Gasparoni, Kap. 3). Diese Prägung muss daher zurückge- 
setzt werden, um wieder einen Zellzustand zu erreichen, aus dem heraus alle Zelltypen des Körpers und sogar ein ganzer Organismus (Totipotenz) gebildet werden können. Mit zunehmenden Teilungen verlieren die Tochterzellen ihr Entwicklungspotenzial und damit ihre Totipotenz bereits in den ersten Tagen nach der Befruchtung.

Pluripotente embryonale Stammzellen (ES-Zellen) kommen im sich entwickelnden Embryo in einem definierten Entwicklungszustand (Blastozystenstadium, 5-6 Tage nach der Befruchtung) vorübergehend (transient) vor. ES-Zellen sind in der Lage, Zellen aller drei Keimblätter des Embryos (Endoderm, Mesoderm und Ektoderm) ${ }^{2}$ zu bilden und können somit in alle über 200 Zelltypen unseres Körpers (einschließlich der Keimbahnzellen: Eizelle bzw. Spermien) ausdifferenzieren (Pluripotenz).

Multipotente adulte (auch somatische oder gewebespezifische) Stammzellen sind dagegen in ihrem Differenzierungspotenzial bereits auf die Bildung spezifischer Organe oder Gewebe festgelegt und können nur Zelltypen des jeweiligen Organsystems oder Gewebes hervorbringen. So differenzieren etwa neuronale Stammzellen in Neurone und andere Zellen des Nervensystems, aber nicht in andere Körperzellen. In unserem Organismus sind multipotente Stammzellen wichtig für die Regeneration und Reparatur von Geweben und Organen. Blutbildende Stammzellen sind bislang der am besten erforschte multipotente adulte Stammzelltyp. Die Transplantation von Blutstammzellen ist ein seit Jahrzehnten etabliertes und gut erprobtes Behandlungsverfahren in der Medizin, z. B. bei Leukämien (Blutkrebs). Ein weiteres wichtiges Beispiel für adulte Stammzellen sind mesenchymale Stammzellen (Bindegewebsstammzellen, auch mesenchymale Stromazellen genannt), die inzwischen zur Behandlung von Knochenund Knorpeldefekten klinisch erprobt werden.

\subsubsection{Künstliche humane Stammzellen - Stammzell-Engineering}

Durch die neuen Erkenntnisse der Entwicklungs- und Molekularbiologie ist es nun in zunehmendem Maße möglich, Stammzellen im Labor künstlich aus adulten Zellen, d. h. aus normalen Körperzellen ohne Stammzelleigenschaften, herzustellen. Dieser Prozess wird als Reprogrammierung bezeichnet. Humane induzierte pluripotente Stammzellen (hiPS-Zellen) sind solche durch Reprogrammierung hergestellte Zellen (Takahashi/Yamanaka, 2006), die z. B. aus Haut- oder Blutzellen relativ einfach und

2 Während der Embryonalentwicklung entstehen im Embryo aus seinen Zellschichten die drei Keimblätter: Ektoderm (Außenschicht), Mesoderm (Mittelschicht) und Endoderm (Innenschicht). Jedes dieser Keimblätter bildet bestimmte Gewebe und Organe aus. So werden etwa Lunge und Darm vom Endoderm gebildet. Wenn ES-Zellen die Zellen aller drei Keimblätter bilden können, so können damit theoretisch Zellen aller Organe und alle Zelltypen unseres Körpers gebildet werden. 
schnell generiert werden können. HiPS-Zellen haben ähnliche Eigenschaften wie hESZellen: HiPS-Zellen sind erstens - ähnlich wie hES-Zellen - immortal, d. h. sie können als hiPS-Zellen unendlich vermehrt werden, sodass hier große Zellmengen hergestellt werden können. Zweitens, können sich hiPS-Zellen in Zellen aller drei Keimblätter, d. h. in alle Zelltypen unseres Körpers, ausdifferenzieren (Pluripotenz). HiPS-Zellen haben das gleiche Genom wie die zur Reprogrammierung verwendeten Körperzellen des Individuums bzw. der Patientin oder des Patienten, inklusive individueller und/oder krankheitsspezifischer Mutationen, sodass hiermit patienten- und krankheitsspezifische Zellen erhalten werden können.

Analog zur iPS-Technologie gibt es heute eine breite Palette an Reprogrammierungsstrategien, von der Reprogrammierung zur Pluripotenz bis zur Umprogrammierung von adulten Zellen zu spezifischen Zellen unter Umgehung des pluripotenten Stadiums („direkte Reprogrammierung“ oder „Transdifferenzierung“). Bei der sogenannten direkten Reprogrammierung werden - ähnlich wie bei der iPS-Reprogrammierung bestimmte Kombinationen von Transkriptionsfaktoren, aber auch RNA, Proteine, spezifische Faktoren und Kulturbedingungen verwendet, um einen neuen Zelltyp herzustellen. So können z. B. Fibroblasten (Bindegewebszellen) direkt zu neuronalen Zellen (Nervenzellen, Neuronen) oder neuralen Stammzellen umprogrammiert werden. Die resultierenden Zellen werden dann als „induzierte Neurone“ bzw. „induzierte neurale Stammzellen“ bezeichnet. Auch induzierte Herzmuskelzellen konnten durch direkte Reprogrammierung mit einer hierfür spezifischen Kollektion an Transkriptionsfaktoren gewonnen werden.

Eine weitere bahnbrechende Entwicklung der letzten Jahre hat einen maßgeblichen Einfluss auf die Stammzellforschung: Genome-Editing mit CRISPR/Cas. Genome-Editing bezeichnet Verfahren, bei denen einzelne DNA-Abschnitte, aber auch größere Genbereiche aus dem Genom gezielt herausgeschnitten oder durch andere DNA-Abschnitte ersetzt werden (siehe auch Fehse, Kap. 6, sowie Fehse et al., Kap. 9). Genome-Editing ist im Zusammenhang mit Stammzellen besonders interessant, da die eingefügte Veränderung der DNA erstens bei der Vermehrung von Stammzellen an die Tochtergenerationen weitergegeben wird und zweitens auch erhalten bleibt, wenn Stammzellen differenzieren und spezialisierte Zelltypen ausbilden.

So werden in einem mehrstufigen Verfahren Stammzellen in Zellkulturen zuerst mit den Methoden des Genome-Editing modifiziert, dann vermehrt und in einem nächsten Schritt in den gewünschten Zelltyp differenziert. Dieser Prozess ist für embryonale und adulte Stammzellen möglich und auch für künstlich hergestellte Stammzelltypen, wie z. B. hiPS-Zellen. Genome-Editing wird u. a. für die Herstellung von Krankheitsmodellen für die Medikamentenentwicklung genutzt. So werden krankheitsspezifische 
Mutationen in hiPS-Zellen zunächst repariert oder eingefügt, sodass hiPS-Zellen mit und ohne Mutationen entstehen. Solche Zellen werden als isogen bezeichnet, da sie bis auf das veränderte Gen genetisch gleich sind. Die hiPS-Zellen mit und ohne Mutationen werden anschließend in die gewünschten Zellen differenziert, die dann für die Erprobung von Medikamenten genutzt werden. Auf diese Weise entstehen patientenspezifische Krankheitsmodelle. Auch die Rückführung von genetisch modifizierten Stammzellen in den Körper im Rahmen der somatischen Gentherapie wird klinisch erprobt.

\subsubsection{Blutbildende/hämatopoetische Stammzellen}

Das hämatopoetische (blutbildende) System (Hämatopoese) ist eines der am besten untersuchten Stammzellsysteme. Die hämatopoetischen Stammzellen (Blutstammzellen) befinden sich im Knochenmark und bilden alle Zelltypen des Blut- und Immunsystems: rote Blutzellen (Erythrozyten) und weiße Blutzellen (Leukozyten) sowie Blutplättchen (Thrombozyten). Die reifen Blutzellen haben nur eine begrenzte Lebensdauer und müssen daher fortlaufend ersetzt und nachgebildet werden. Das blutbildende System ist dabei hierarchisch aufgebaut: Hämatopoetische Stammzellen stehen an der Spitze einer Differenzierungskaskade, die über begrenzt teilungsfähige Vorläuferzellen bis hin zu den verschiedenen reifen Blutzelltypen führt. Einzelzellanalysen (siehe Walter/ Schickl, 2019, und Fehse et al., Kap. 9) haben nun gezeigt, dass das Potenzial von hämatopoetischen Stammzellen, einen bestimmten reifen Blutzelltyp zu ergeben, bereits auf der Ebene der Stammzellen angelegt sein kann. Auch ist die Differenzierung von hämatopoetischen Stammzellen zu reifen Blutzellen eher ein dynamischer und kontinuierlicher Entwicklungsprozess als - wie bisher angenommen - ein Durchlaufen diskreter Entwicklungsstufen (Laurenti/Göttgens, 2018). Ist die Entwicklung von Blutstammzellen zu reifen Blutzellen gestört, so kann dies zu Leukämien (Blutkrebs) führen.

Blutstammzellen werden bereits seit vielen Jahren routinemäßig in der Klinik für die Stammzelltransplantation genutzt (Müller-Röber et al., 2015). Auch werden erste klinische Studien mit durch CRISPR/Cas-Editing modifizierten Blutstammzellen bei der Sichelzellanämie durchgeführt. Allerdings ist die Vermehrbarkeit von Blutstammzellen im Labor unter Beibehaltung des multipotenten Stammzellpotenzials zurzeit aus noch nicht verstandenen Gründen begrenzt. Diese Fragestellung wird nach wie vor intensiv erforscht (siehe unten). 


\subsubsection{Stammzellen aus Nabelschnurblut}

Stammzellen können nach der Geburt aus dem Blut der Nabelschnur entnommen und für einen späteren Einsatz eingefroren werden. Nabelschnurblutstammzellen können entweder kostenpflichtig für den Eigenbedarf bei einer privaten Blutbank eingelagert oder aber an eine gemeinnützige öffentliche Blutbank gespendet werden. Nabelschnurblutstammzellen enthalten $u$. a. hämatopoetische Stammzellen, die zur Behandlung von Bluterkrankungen transplantiert werden. Ein Vorteil der Nabelschnurstammzellen gegenüber adulten Stammzellen von Knochenmarkspenderinnen oder Knochenmarkspendern ist eine bessere Immunverträglichkeit des Transplantats. Nachteilig ist jedoch, dass im Nabelschnurblut nur eine begrenzte Anzahl von Stammzellen vorhanden ist und diese bisher nicht effizient vermehrt werden können. Es ist daher ein erklärtes Ziel der Stammzellforschung, die Nutzbarkeit von Nabelschnurblutstammzellen für die medizinische Therapie zu verbessern.

\subsubsection{Mesenchymale Stammzellen}

Mesenchymale Stammzellen (Bindegewebsstammzellen, auch mesenchymale Stromazellen genannt) sind multipotente Stammzellen und können in die verschiedenen Zelltypen des Körperskeletts (Knorpel, Knochen, Sehnen, Bänder und Fettgewebe) differenzieren. Mesenchymale Stammzellen kommen wie hämatopoetische Stammzellen im Knochenmark vor, aber auch im Nabelschnurblut oder Fettgewebe, und können so relativ einfach gewonnen werden. Ihre therapeutische Anwendung ist bereits - ähnlich wie bei den Blutstammzellen - weit vorangeschritten und umfasst in erster Linie die Behandlung von Knochen- und Knorpelschäden, aber auch die Behandlung von Immunerkrankungen. Das Potenzial mesenchymaler Stammzellen zur Therapie weiterer Erkrankungen wird derzeit in einer Vielzahl von klinischen Studien erforscht. ${ }^{3}$

\subsubsection{Neurale Stammzellen}

Neurale Stammzellen sind multipotente Stammzellen des Nervensystems und können in die verschiedenen spezialisierten neuralen Zelltypen differenzieren, wie z. B. in Neurone, Astrozyten und Oligodendrozyten. Während der Embryonalentwicklung generieren neurale Stammzellen die verschiedenen Zelltypen des zentralen und peripheren Nervensystems (Neurogenese). Im erwachsenen Organismus befinden sich die

3 Siehe unter: https://clinicaltrials.gov [23.06.2021] und https://www.clinicaltrialsregister.eu [23.06.2021]. 
neuralen Stammzellen hauptsächlich in zwei hochspezialisierten diskreten Regionen des Gehirns, der subventrikulären und der subgranulären Zone. Hier sind neurale Stammzellen wichtig für das Aufrechterhalten der Gehirnfunktion und die Regeneration der Nervenzellen im Gehirn z. B. nach Verletzungen.

Neurale Stammzellen werden im Zusammenhang mit neurodegenerativen Erkrankungen, wie der Parkinson-Krankheit und Alzheimer-Krankheit, besonders intensiv erforscht. Erklärtes Ziel dieser Studien ist, die Mechanismen der Krankheitsentstehung und -progression zu verstehen und die Erkrankungen mittels einer zellbasierten Therapie behandeln zu können. Die Gewinnung von neuralen Stammzellen aus Gehirngewebe von Patientinnen und Patienten ist schwierig und oft auch aus ethischen Gründen nicht möglich, sodass hier andere Wege beschritten werden: Erstens, die Differenzierung von pluripotenten Stammzellen (hES-Zellen und hiPS-Zellen) in neurale Stammzellen und weiter in die gewünschten spezialisierten neuralen Zelltypen; zweitens die Transdifferenzierung von normalen Körperzellen (somatischen Zellen, wie z. B. Fibroblasten) direkt zu neuronalen Zellen oder zu neuralen Stammzellen (induzierte Neurone bzw. induzierte neurale Stammzellen; siehe oben), die dann weiter in die gewünschten spezialisierten neuralen Zelltypen differenziert werden. Dies geschieht oft im Rahmen dreidimensionaler Zellaggregate (Spheroide, Organoide; siehe unten), da so die natürliche Umgebung neuraler Zellen besser abgebildet wird. Auch wird die Wirksamkeit der Transplantation von aus hES-Zellen abgeleiteten Neuronen bei der Parkinson-Krankheit in ersten klinischen Studien erprobt. ${ }^{4}$

\subsection{Organoide}

\subsubsection{Organoide aus adulten und pluripotenten Stammzellen}

Organoide können sowohl aus gewebespezifischen adulten Stammzellen als auch aus pluripotenten Stammzellen (hES-Zellen oder hiPS-Zellen) hergestellt werden. Die Verwendung von adulten bzw. pluripotenten Stammzellen für Organoide erfordert unterschiedliche Kulturbedingungen, was mit jeweiligen Vor- und Nachteilen einhergeht.

Adulte Stammzellen sind bereits in ihrem Differenzierungspotenzial auf die Bildung spezifischer Organe oder Gewebe festgelegt und können nur Zelltypen des jeweiligen Organsystems oder Gewebes hervorbringen (siehe oben). Bei der Gewinnung von Organoiden aus adulten Stammzellen geht es daher darum, die normale Entwicklung des jeweiligen Organsystems in der Kulturschale nachzuvollziehen, indem die Umgebung des

Siehe unter: https://clinicaltrials.gov [23.06.2021]. 
Körpers nachgebildet wird. Dies geschieht z. B. durch die Verwendung von spezifischen Kulturbedingungen oder die Zugabe einer dreidimensionalen Gerüststruktur (Matrix) und von speziellen Signalstoffen. So erfordert die Differenzierung einer Darmepithelstammzelle zu Darmepithel in unserem Körper eine bestimmte dreidimensionale Umgebung und spezielle Signalstoffe. Entsprechend führt die Zugabe dieser Signalstoffe zu Darmepithelstammzellen in der Kulturschale mit dreidimensionaler Matrix - wie in unserem Körper - zur Bildung von neuem Darmepithel, einem Darmorganoid.

Die Bildung von Organoiden aus pluripotenten Stammzellen folgt einem ähnlichen Prinzip: auch hier wird die natürliche Umgebung der Stammzelle in der Kulturschale nachgeahmt. Pluripotente Stammzellen haben ein sehr viel breiteres Spektrum an Differenzierungsmöglichkeiten als adulte Stammzellen, sodass bei ihrer Entwicklung zu Organoiden eine ganze Reihe von Entwicklungsschritten durchlaufen werden müssen. Dafür werden die Kenntnisse der Embryonalentwicklung genutzt: In aufeinander folgenden Schritten werden definierte Signalstoffe hinzugegeben, die auch im Körper dafür sorgen, dass die pluripotenten Stammzellen eine bestimmte Entwicklungsrichtung einschlagen. So wie aus Stammzellen in einem frühen Embryo erst nach vielen Entwicklungsschritten eine Darmwand wird, wird auch in der Zellkultur erst nach einigen Wochen aus pluripotenten Stammzellen eine Darmstammzelle, die dann ein Darmorganoid hervorbringt.

Die Verwendung von adulten bzw. pluripotenten Stammzellen unterscheidet sich nicht nur in der Kultivierung der Organoide, sondern auch in ihren jeweiligen Vorund Nachteilen. Die Organoide aus adulten Stammzellen können bisher nur aus Epithel generiert werden und bleiben auch nur Epithel; so generiert eine adulte Darmepithel-Stammzelle eben Darmepithel, aber kein Bindegewebe. Bei den aus pluripotenten Stammzellen abgeleiteten Organoiden ist das anders: Sie können auch andere Zelltypen enthalten, sind komplexer und heterogener - z. B. enthalten die so gewonnenen Darmorganoide auch Bindegewebe. Pluripotente Stammzellen können dadurch zu so unterschiedlichen Organoiden wie Darmorganoiden, Hirnorganoiden und daneben auch zu Embryoiden differenziert werden. Hirnorganoide und Embryoide werden dagegen aus adulten Stammzellen bisher nicht erhalten.

Allerdings können derzeit noch nicht alle Organe in Form von Organoiden nachgebildet werden, und die bereits entwickelten Organoide bilden nicht immer alle Zelltypen eines Organs ab. Beispielsweise ist ein Darm umgeben von Blutgefäßen und Nerven, die aber im Organoid nicht enthalten sind. Abhängig von der Fragestellung kann das die Übertragbarkeit von Forschungsergebnissen auf ein im Körper befindliches Organ erheblich einschränken. Großes Potenzial hat daher die nächste Generation von Organoiden, die komplexer und reifer sein werden und die jeweiligen Organe besser 
nachahmen können, z. B. dadurch, dass sie Blutgefäße, Immunzellen oder Nervengewebe enthalten. Neuere Forschungsansätze untersuchen darüber hinaus Wechselwirkungen zwischen Organen mittels der Multi-Organ-on-a-Chip-Technologie, bei der unterschiedliche Organoide auf einem Chip miteinander verknüpft werden (z. B. Leber-, Nieren- und Lungenorganoide). Zudem können verschiedene Organoide, beispielsweise Organoide verschiedener Hirnareale, zu sogenannten „Assembloiden“ zusammengesetzt werden. Auf diese Weise kann dann auch das Zusammenspiel verschiedener Zelltypen und Regionen bei der Organentwicklung und bei Multiorganerkrankungen untersucht werden.

\subsubsection{Stammzellen und Organoide als Krankheitsmodelle}

Stammzellen und Organoide ermöglichen die wissenschaftliche Erforschung der menschlichen Entwicklung und Physiologie, der Organentwicklung und -regeneration in einem zuvor nicht gekannten Ausmaß und mit hoher Präzision. Dies gilt sowohl für normale physiologische, also „gesunde“ Bedingungen wie auch für pathologische, also „krankhaft" veränderte Bedingungen. Zudem hat sich das Spektrum der Organe, die mit Organoiden erforscht werden können, in den letzten Jahren kontinuierlich erweitert. Es ist davon auszugehen, dass sich dieser Trend in Zukunft fortsetzen wird.

Organoide aus gesunden menschlichen Zellen enthalten viele der in einem Organ vorkommenden Zelltypen und weisen eine stabile Genotyp-Phänotyp-Beziehung sowie Aspekte der Architektur, Physiologie und Funktion des menschlichen Organs auf. Aus diesen Gründen können viele komplexe Vorgänge gut mit Organoiden erforscht werden. In der Grundlagenforschung gehören dazu z. B. Untersuchungen der menschlichen Embryonalentwicklung, Organentwicklung (Organogenese) und der Aufrechterhaltung der Organfunktion.

Stammzellen und Organoide werden sowohl aus gesundem als auch aus erkranktem Gewebe hergestellt und eignen sich daher als Krankheitsmodelle für die Erforschung einer breiten Palette von Erkrankungen: genetisch bedingter Krankheiten mit vererbbaren Mutationen, onkologischer Erkrankungen mit erworbenen Mutationen und auch von Stoffwechsel- und Infektionskrankheiten. Im Rahmen der personalisierten Medizin werden patienteneigene autologe Organoide und aus Stammzellen abgeleitete Zellen dazu genutzt, verschiedene Medikamente vor einer Verabreichung zu testen, und so das für die Patientin bzw. den Patienten am besten geeignete Medikament auszuwählen. Im Fall der Mukoviszidose, einer angeborenen Stoffwechselerkrankung, werden aus Patientenzellen entwickelte Organoide bereits zu diesem Zweck genutzt. Diese prognostische Anwendung ist bereits Teil des niederländischen Gesundheitssystems. 
Ein besonderes Interesse gilt Organoiden aus humanen pluripotenten Stammzellen (hES-Zellen und iPS-Zellen). Die Gründe hierfür sind: erstens der mangelnde Zugang zu primärem gesundem und erkranktem Gewebe von Patientinnen und Patienten und zweitens das pluripotente und damit besonders breite Differenzierungspotenzial von hES-Zellen und iPS-Zellen und damit die Möglichkeit, verschiedene Organoidtypen aus ein und derselben Ausgangszellpopulation herzustellen.

Hierbei werden Blut- oder Bindegewebszellen von Patientinnen und Patienten in hiPS-Zellen reprogrammiert. Diese patienten- und krankheitsspezifischen hiPS-Zellen enthalten das individuelle genetische Profil der Patientin bzw. des Patienten und die die Erkrankung auslösenden Mutation(en) wie auch andere mit der Erkrankung assoziierte Mutationen. Diese hiPS-Zellen werden zunächst vermehrt und dann in den gewünschten Zelltyp und/oder das gewünschte Organoid differenziert, z. B. in neurale Zellen und Hirnorganoide, und für die Medikamententestung, z. B. von Psychopharmaka, eingesetzt. Die gleichen hiPS-Zellen können auch in Herzmuskelzellen und Leberzellen und die entsprechenden Organoide differenziert werden, um die mögliche Toxizität des Medikaments für Herzmuskelzellen und die Metabolisierung des Medikaments durch Leberzellen auf dem für die Patientin bzw. den Patienten spezifischen genetischen Hintergrund zu bestimmen. Dieser Bereich der personalisierten Medizin wird als Begleitdiagnostik bezeichnet und erlebt gegenwärtig eine rasante Entwicklung.

Zusätzlich werden Stammzellen und Organoide in „lebenden Biobanken“ erfasst und eingelagert. ${ }^{5}$ Eingelagerte Stammzellen können jederzeit aufgetaut und erneut vermehrt oder auch in Organoide differenziert werden. Auch Organoidbiobanken wurden bereits weltweit für viele Organe aufgebaut, beispielsweise gibt es Biobanken von Darmorganoiden, Leberorganoiden und Organoiden der Nieren- und Harnwege. Sie können jeweils Organoide von mehreren Hundert Patientinnen und Patienten umfassen. Je nach wissenschaftlicher bzw. medizinischer Fragestellung können entweder spezielle Gruppen von Organoiden aufgetaut werden oder ganze Biobanken. Diese Banken haben eine besondere Bedeutung für die Medikamententestung: Die gelagerten Organoide können verwendet werden, um nach neuen Medikamenten zu suchen (Screening) oder die Bedeutung eines Medikaments für eine Gruppe von Patientinnen und Patienten zu testen (patientenspezifische Wirksamkeit); sie können auch für die Toxikologie eingesetzt werden.

5 Siehe unter: https://ebisc.org [23.06.2021] und https://huborganoids.nl [23.06.2021]. 
Abbildung 1: Einsatzmöglichkeiten von aus Stammzellen abgeleiteten Organoiden ${ }^{6}$

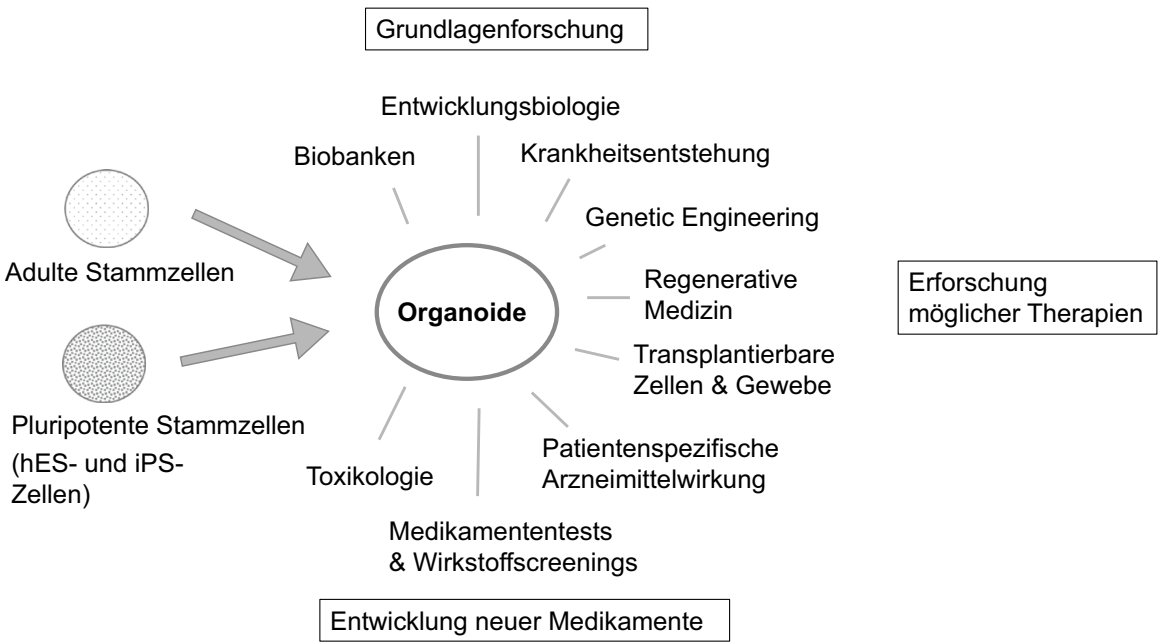

\subsubsection{Stammzellen und Organoide für die regenerative Medizin}

Die Verwendung von Stammzellen in der regenerativen Medizin ist bei einigen Stammzelltypen, wie den Blutstammzellen, bereits klinische Routine (siehe oben). Gleichzeitig ist die Entwicklung weiterer Stammzelltypen für die Transplantation und die regenerative Medizin weiterhin ein zentrales Ziel der Stammzellforschung. Mit den Fortschritten in der Organoidtechnologie rückt die Transplantation von Organoiden oder von aus Organoiden abgeleiteten Zellen in der Zellersatz- und regenerativen Therapie in den Fokus. Dabei sind Transplantate sowohl aus eigenem (autologem) als auch fremdem (allogenem) Material denkbar. Die Organoidtechnologie würde hier dazu genutzt werden, den gewünschten Zelltyp in der für eine Transplantation notwendigen Reife und Menge herzustellen.

Diese Entwicklungen sind bei der Transplantation des retinalen Pigmentepithels zur Behandlung der altersbedingten Makuladegeneration (AMD) besonders weit vorangeschritten und werden weltweit in klinischen Studien untersucht. ${ }^{7}$ AMD ist eine Erkrankung der Augennetzhaut und mit 32 \% der Neuerblindungen die häufigste Ursache für Erblindung im Erwachsenenalter. Sie betrifft in Deutschland schätzungsweise 2 Millionen Menschen. ${ }^{8}$

6 Die beiden Abbildungen stammen von Lilian Marx-Stölting.

7 Siehe unter: https://clinicaltrials.gov [23.06.2021].

8 Siehe unter: https://de.wikipedia.org/wiki/Makuladegeneration [23.06.2021]. 
Bedeutsam in diesem Kontext ist dann auch, dass Stammzellen mit den Methoden des Genome-Editing besonders leicht modifiziert werden können, um z. B. (die) krankheitsauslösende(n) Mutation(en) zu korrigieren (siehe oben). Die auf diese Weise „reparierten" Stammzellen sind dann das Ausgangsmaterial für gesunde Organoide für die Transplantation. Erste Experimente im Tiermodell lieferten bereits vielversprechende Ergebnisse; weitere Studien zur Funktionalität von Organoiden in vivo sind jedoch nötig. Auch ist es bis zur Transplantation eines aus Stammzellen und/oder Organoiden abgeleiteten transplantierbaren Organs noch ein weiter Weg.

In diesem Zusammenhang von besonderer Bedeutung sind sogenannte ungeprüfte Stammzelltherapien, die in zunehmendem Maße über das Internet international kommerziell angeboten werden. Ungeprüfte Stammzelltherapien sind stammzellbasierte Therapien, die nicht im Rahmen klinischer Studien auf ihre Sicherheit und Wirksamkeit geprüft wurden und deren Wirkstoffe daher keine behördliche Zulassung haben. oft werden hier stammzellbasierte Therapien mit ein und demselben Verfahren für eine ganze Palette von schwersten und bisher nicht oder nur ungenügend therapierbaren Erkrankungen angeboten. Es fehlen Informationen zu den verwendeten Zellen, ihrer Anwendung und ihrer Wirkungsweise. Diese Angebote beziehen sich auf das stammzelleigene Regenerationspotenzial und wollen Menschen in häufig verzweifelten Lebenssituationen ansprechen. Die sich aus ungeprüften Stammzelltherapieangeboten ergebende Problematik wird in zunehmendem Maße international und national von Stammzellforscherinnen und -forschern wahrgenommen und diskutiert. Die Internationale Gesellschaft für Stammzellforschung (International Society for Stem Cell Research, ISSCR), das Deutsche Stammzellnetzwerk (German Stem Cell Network, GSCN) und das Stammzellnetzwerk NRW haben webbasierte Informationsplattformen eingerichtet, auf denen sich Patientinnen und Patienten über zugelassene Stammzelltherapien und Risiken ungeprüfter Stammzelltherapien informieren können. ${ }^{9}$

\subsubsection{Embryoide}

In den letzten Jahren wurden aus Maus und humanen pluripotenten Stammzellen komplexe, organisierte Strukturen gebildet, die sehr frühen Stadien von Embryonen ähnlich sind. Die Forschung an Mausstammzellen ist dabei erheblich weiter als die an humanen Stammzellen. Manche Forscherinnen und Forscher gehen davon aus, dass es in absehbarer Zeit möglich sein wird, Strukturen zu schaffen, die nicht mehr von

9 Siehe unter: https://de.gscn.org/Portals/0/Dokumente/Material/isscr_germanguidelines.pdf [23.06.2021], https://de.gscn.org/de/ZUSTAMMZELLEN/Patienteninformationen.aspx [23.06.2021] und https://www.stammzellen.nrw.de/informieren/patienteninformation [23.06.2021]. 
einem Embryo unterscheidbar sind. Die neuen Entitäten werden u. a. als „synthetische Embryonen“, „Embryoide“ oder auch „Blastoide“ bezeichnet. Diese sollten nicht verwechselt werden mit den schon lange bekannten „Embryoid Bodies, EBs“, die als undifferenzierte Aggregate von pluripotenten Stammzellen weniger komplex und organisiert sind, und eine Vorstufe von Embryoiden bilden können. Aufgrund der Ähnlichkeit von Embryoiden mit menschlichen Embryonen werden diese derzeit in Übereinstimmung mit den rechtlichen Regularien vieler Länder zu menschlichen Embryonen nicht länger als 14 Tage kultiviert. Es ist aber eine offene Frage, wie Embryoide ontologisch einzustufen sind (so wie menschliche Embryonen oder anders?), wie sie entsprechend bezeichnet werden sollten und welcher normative Status ihnen zugeschrieben werden sollte (siehe dazu weiter unten).

Diese offenen ethischen Fragen müssen auf der Basis einer interdisziplinären und gesamtgesellschaftlichen Debatte diskutiert werden, um politisch wie rechtlich konsensfähige Lösungen zu finden.

\subsection{Ethische und rechtliche Einordnung der Forschung an Stammzellen und Organoiden}

Abbildung 2: Ethische Aspekte im Bereich Organoide

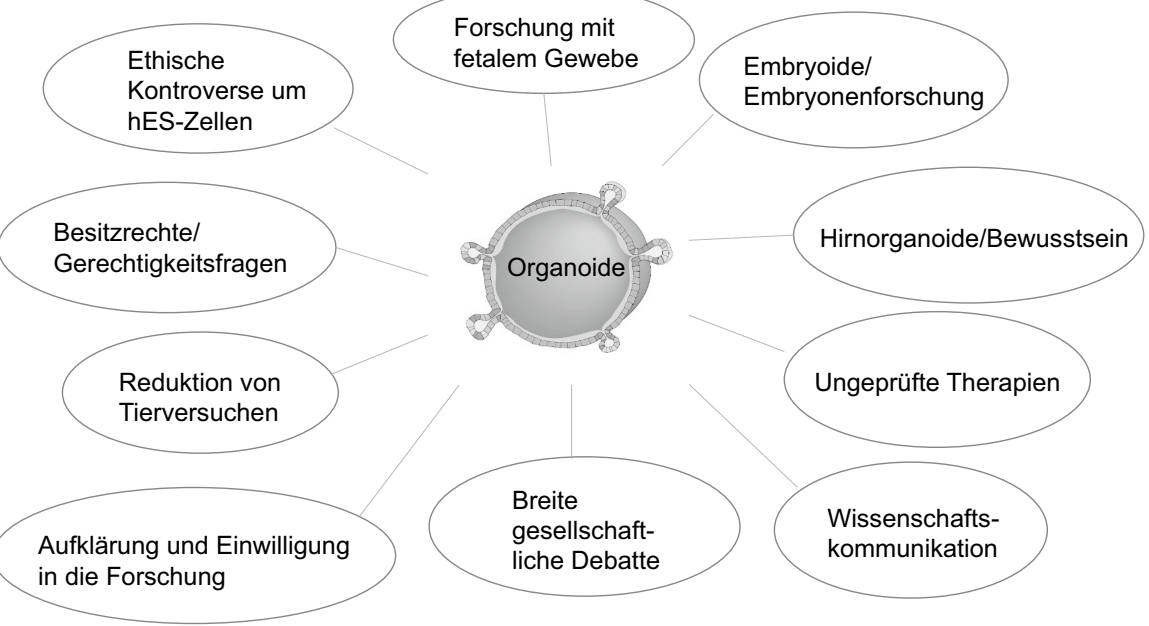




\subsubsection{Adulte und pluripotente Stammzellen}

Adulte Stammzellen, wie Blutstammzellen und mesenchymale Stammzellen, haben in der Öffentlichkeit ein positives Image und sind ethisch und rechtlich wenig umstritten. Dies gilt auch für die Forschung an hiPS-Zellen. Die Forschung an hES-Zellen hingegen wird in Deutschland aufgrund der Herkunft der Zellen als ethisch problematisch angesehen und die Gewinnung von hES-Zellen ist in Deutschland durch das Embryonenschutzgesetz (ESchG) verboten. Die Forschung an im Ausland generierten und nach Deutschland importierten hES-Zellen ist zwar seit dem 01.01.2002 nach dem Stammzellgesetz (StZG) zulässig, aber nur in begründeten Ausnahmefällen, und unter strengen Voraussetzungen und nur für Forschungszwecke.

Die in Deutschland gängige Annahme, dass die Forschung mit hiPS-Zellen eine Alternative zur Forschung mit hES-Zellen darstelle, hat sich in der Praxis allerdings nicht bestätigt. Zwar hat sich der Forschungsfokus seit der Entdeckung von hiPS-Zellen verschoben und hiPS-Zellen sind in Deutschland und auch weltweit in vielen Fällen zum primären Forschungsobjekt geworden. Allerdings sind hES-Zellen zum einen oft auch noch alleiniger Forschungsgegenstand. Zum anderen werden hiPS-Zellen und hES-Zellen häufig gemeinsam untersucht, da hES-Zellen international weiterhin als Referenz für die Pluripotenz gelten, u. a. weil das Forschungsgebiet der hiPS-Zellen jünger ist und hES-Zellen besser erforscht sind. Die hiPS-Zell-Forschung ist daher nach wie vor auf die hES-Zell-Forschung angewiesen und wird dies auch noch lange sein.

Des Weiteren sind inzwischen neuere, sogenannte „naive“ hES-Zellen verfügbar, die einen im Vergleich zu konventionellen, sogenannten ,primed“ hES-Zellen weniger weit differenzierten Zustand haben. Eine Forschung an naiven hES-Zellen ist in Deutschland allerdings ebenfalls durch das StZG verboten, da naive hES-Zellen erst nach dem geltenden Stichtag, bis zu dem der Stammzellimport zulässig ist (01.05.2007), beschrieben und gewonnen wurden. Auch neuere hES-Zellen für die klinische Anwendung, sogenannte „clinical-grade“ hES-Zellen, wurden nach dem Stichtag gewonnen, wodurch ihr Import sowie ihre Verwendung in Deutschland ebenfalls verboten sind.

Inzwischen mehren sich die Stimmen, die eine grundlegende Revision des Stammzellgesetzes fordern. So wird die Stichtagsregelung ebenso kritisiert wie das aufwendige Genehmigungsverfahren. Beides ist auch verfassungsrechtlich problematisch, da die im Grundgesetz verankerte Forschungsfreiheit eingeschränkt wird. Auch hat in den vergangenen Jahren die Anzahl klinischer Studien im Ausland deutlich zugenommen, bei denen die therapeutische Wirksamkeit von aus hES-Zellen abgeleiteten Zellen bei schwerwiegenden Erkrankungen untersucht wird. Vor diesem Hintergrund erscheint die Begrenzung der Verwendung von hES-Zellen in Deutschland allein auf die Forschung nicht mehr haltbar. Der Gesetzgeber in Deutschland sollte, schon wegen des 
Grundrechts auf Leben und körperliche Unversehrtheit, Patientinnen und Patienten im Inland diese künftigen Therapien nicht vorenthalten.

\subsubsection{Organoide und Embryoide}

Organoide aus adulten Stammzellen sind ähnlich wie die adulten Stammzellen selbst ethisch und rechtlich wenig umstritten. Das liegt wahrscheinlich auch daran, dass ethisch und rechtlich sensible Strukturen wie das Gehirn oder ein Embryo nicht mit ihnen nachzubilden sind. Diskutiert wird aber der Umgang mit Spendermaterial und -daten auch im Rahmen von Biobanken.

Bei Organoiden aus pluripotenten Stammzellen stehen Hirnorganoide, Assembloide, die Erzeugung von Mensch-Tier-Chimären ebenso wie der Umgang mit Embryoiden im Fokus der Diskussion. Aktuell sind Hirnorganoide noch weit von einem komplexen, menschlichen Gehirn entfernt. Aber es wurde vielfach die Frage aufgeworfen, ob in Zukunft komplexere Hirnorganoide oder miteinander fusionierte Organoide verschiedener Hirnareale (sogenannte „Assembloide“) ein Bewusstsein entwickeln könnten, und wenn, wie ein solches messbar wäre und welche ethisch-rechtlichen Schutzansprüche dann ggf. daraus abzuleiten wären (zur rechtlichen Einordnung von Hirnorganoiden siehe Taupitz, Kap. 15). Aus derzeitiger Sicht ist den jetzigen Hirnorganoiden die Kapazität zu einer solchen Bewusstseinsentwicklung oder Schmerzempfindung deutlich abzusprechen.

Werden humane Hirnorganoide in lebende Säugetiere (z. B. Ratten, Mäuse, ggf. auch größere Säugetiere) transplantiert, also „Mensch-Tier-Chimären“ erzeugt, wird die Frage aufgeworfen, inwieweit dies die Kapazitäten hinsichtlich Intelligenz und Leidensfähigkeit verändern könnte und welche Konsequenzen daraus zu ziehen wären. Mit wachsender Komplexität der Hirnorganoide, Assembloide und Chimären, müssen in Zukunft diese ethischen Fragen weiter diskutiert werden.

Bei den Embryoiden wird international anhand der Komplexität und dem Potenzial differenziert. Die ISSCR unterscheidet in den 2021 herausgegebenen Leitlinien zum Umgang mit Stammzellen, Organoiden und Embryomodellen zwei Typen von Embryoiden. ${ }^{10}$ Auf der einen Seite stehen Strukturen, die alle Gewebe enthalten bzw. hervorbringen können, die aus der befruchteten Eizelle entstehen, dem sogenannten „Konzeptus“. Ein kompletter Konzeptus enthält den Embryo, die Plazenta und andere extraembryonale Gewebe. Die Modelle enthalten entsprechend embryonale und extra-

10 Siehe unter: www.isscr.org/policy/guidelines-for-stem-cell-research-and-clinical-translation [23.06.2021]. 
embryonale Zellen, die prinzipiell das Potenzial haben, sich zu einem Organismus zu entwickeln. Blastoide (Modelle der Blastozyste) sind ein Beispiel für solche kompletten Modelle. Auf der anderen Seite stehen Strukturen, die nur Teile des Konzeptus enthalten, also beispielsweise nur die Plazenta oder nur den Embryo. Die zweite Gruppe von Strukturen ist nach derzeitigem Erkenntnisstand grundsätzlich nicht in der Lage, sich zu einem intakten, kompletten Konzeptus zu entwickeln. Gastruloids (Modelle der Gastrula, ein Stadium nach dem Blastozystenstadium) sind ein Beispiel für solche inkompletten Modelle.

Die ISSCR empfiehlt, in der ethischen Beurteilung zwischen den kompletten und inkompletten Modellen zu unterscheiden. Forschung an Modellen, die nur verwendet werden, um einzelne anatomische Strukturen zu verstehen, nicht aber, um die Entwicklung des kompletten Konzeptus nachzuvollziehen, sollten demnach initial durch lokale Ethikkommissionen geprüft werden und könnten, müssten aber nicht, durch einen Begutachtungsprozess begleitet werden. Erzeugung und Forschung an Modellen, die den kompletten Konzeptus abbilden, sollte hingegen durch entsprechende Kommissionen begleitet werden und sollte wichtige wissenschaftliche Ziele verfolgen, die nicht durch Forschung an alternativen Modellen erreicht werden können. Der Transfer von Modellen, die den kompletten Konzeptus abbilden, in einen menschlichen oder tierischen Uterus ist generell als unethisch abzulehnen.

Obwohl es in Deutschland keine spezifischen Rechtsregeln zur Herstellung und Verwendung von Organoiden gibt, werden sie doch sowohl vom Verfassungsrecht als auch vom einfachen Recht erfasst (siehe Taupitz, Kap. 15). Bezüglich der Herkunft des Ausgangsmaterials können Organoide insbesondere unter das Stammzellgesetz fallen, das den Import und die Verwendung von hES-Zellen regelt.

Bei Embryoiden hängt die rechtliche Einordnung insbesondere davon ab, ob sie als menschliche Lebewesen mit einer Entwicklungsfähigkeit ähnlich der des menschlichen Embryos eingestuft werden können. Damit könnte ihnen dann unter Umständen Menschenwürde und Lebensschutz zuzuweisen sein. Ob sie schon nach bereits geltendem Recht vom Embryonenschutzgesetz erfasst werden, ist völlig ungeklärt. Embryoide werden ohne natürliche Befruchtung und ohne das Ziel der Erzeugung von Nachkommen erhalten. Auch wird ihre Entwicklung in einem sehr frühen Stadium beendet. Damit ist rechtspolitisch zu überlegen, ob den menschlichen Embryoiden ein so starker rechtlicher Schutz wie dem natürlichen menschlichen Embryo zugesprochen werden sollte. 


\subsection{Literaturverzeichnis}

Bartfeld, S. et al. (2020a): Organoide - Ihre Bedeutung für Forschung, Medizin und Gesellschaft. Nomos, Baden-Baden.

Bartfeld, S. et al. (2020b): Organoide in Forschung und Anwendung: eine Einführung. In: Bartfeld, S. et al. (Hrsg.): Organoide - Ihre Bedeutung für Forschung, Medizin und Gesellschaft. Nomos, Baden-Baden: 44-64.

Laurenti, E./Göttgens, B. (2018): From haematopoietic stem cells to complex differentiation landscapes. In: Nature 553(7689): 418-426.

Müller-Röber, B. et al. (2015): Dritter Gentechnologiebericht. Analyse einer Hochtechnologie. Nomos, Baden-Baden.

Takahashi, K./Yamanka, S. (2006): Induction of pluripotent stem cells from mouse embryonic and adult fibroblast cultures by defined factors. In: Cell. 126(4): 663-676.

Walter, J./Schickl, H. (2019): Einzelzellanalyse in Forschung und Medizin. Eine Stellungnahme der interdisziplinären Arbeitsgruppe Gentechnologiebericht. BBAW, Berlin.

Zenke, M. et al. (2018a): Stammzellforschung - Aktuelle wissenschaftliche und gesellschaftliche Entwicklungen. Nomos, Baden-Baden.

Zenke, M. et al. (2018b): Aktuelle Entwicklungen der Stammzellforschung: eine Einleitung. In: Zenke, M. et al. (Hrsg.): Stammzellforschung - Aktuelle wissenschaftliche und gesellschaftliche Entwicklungen. Nomos, Baden-Baden: 35-52. 